\title{
SHOULD THE DECISION-MAKING PROCESS OF REIMBURSING MEDICINES BE IMPROVED?
}

Pharmaceutical sales totaled an estimated USD 700 billion in OECD countries in 2011, approximately one-fifth of total healthcare spending (5). In the past, pharmaceutical expenditure has increased faster than total healthcare expenditure in many countries. The increase in pharmaceutical expenditure has slowed down in recent years due to the economic recession, patent expirations of important blockbusters, and costcontainment measures already taken. However, pharmaceutical expenditure still continues to rise, albeit at a reduced rate. Alongside an increased consumption of medicines, an important reason for growing costs is the adoption of new-and typically more expensive - products. To ensure that pharmaceutical expenditure does not become excessive and that patients have access to medicines they need, most countries now weigh up what products are to be included in the reimbursement system and at what price. Both the criteria to assess products and the processes to make reimbursement decisions vary from country to country and so far there have been no analytically oriented comparison studies.

In this issue of the Journal, Franken et al. (2) describe and compare the reimbursement decision processes in five European Union (EU) countries: Austria, Belgium, France, the Netherlands, and Sweden. Descriptions of reimbursement systems of different countries have previously been available, but Franken and her colleagues study the issue in greater depth. The authors note that the small number of countries included is a limitation of their study. In the knowledge of how laborious it is to learn to understand foreign reimbursement systems, the number of countries can be regarded as adequate, and little benefit would be gained from analyzing more systems.

The study shows that there are many similarities between the countries. Some of the similarities, like the time frame of the decisions, application-based processes, and the possibility of the applicant to appeal, are based on EU legislation. Similarities can in part be the result of regular communication between reimbursement authorities in the EU countries. Joint meetings and shared information may have contributed to the diffusion of actions to other countries. However, there are also differences between the countries. Some of them are small but others more noteworthy. It would be very interest- ing to know the importance of the differences identified, for example, do they result in diverse reimbursement decisions? The present study does not compare reimbursement decisions which the study countries have made, but the authors propose to do that in future studies. Some evidence is available in the published research literature showing that decisions made in different countries can be quite similar despite differences in the process. Austria, Finland, Norway, and Sweden all restricted the reimbursement of expensive statins after 2000. (3) Likewise, when the first biological product for rheumatoid arthritis came onto the market its reimbursement was restricted in many European countries (4). It has, however, been reported that patients' access to medicinal products varies from country to country (6).

The study shows that the transparency of the decision making remains a problem despite countries having tried to improve it. No formal hierarchy exists for the reimbursement criteria used, and the weight of each criterion is not determined. The message of the authors is the same as that which has often been highlighted by the pharmaceutical industry.

As a solution to the lack of transparency, the research group introduces in this Journal issue a framework they have developed to improve the transparency of reimbursement decisions (1). The researchers approach the problem with five questions and accompanying relevant criteria to be considered when addressing the questions. The questions are those that are under consideration in many countries even if they are not always recorded explicitly. Is there a need for a new therapy? Are we prepared to pay for a new therapy? Are we prepared to pay for this new treatment? If yes, are we prepared to pay more than for already available treatments? How much are we prepared to pay? The actual questions could be: Do we need better treatment for asthma? Are we prepared to pay for smoking cessation? Are we prepared to pay for still another antidepressant and how much? How much more are we prepared to pay for a product to be administered once a month instead of once a day?

In practice, many of the questions are not easy to answer, and without piloting the tool it is impossible to say how useful and feasible it might be in formal decision making. Reimbursement 
decision making is challenging - at least when new products are concerned - especially due to the uncertainty of the evidence regarding the health effects and cost of treatments and the strict time frame. In the EU countries, a decision must be made in 180 days, and it has been proposed that even this length of time be shortened. Furthermore, the authors point out that the proposed tool is not adequate as such. Weights of each criterion remain to be determined by the decision makers, which should perhaps be addressed case by case. For example, the tool does not give us an answer on how much an orphan drug should cost or what kind of clinical evidence is enough when the disease is rare and a randomized clinical trial is not possible.

Determining weights for the criteria is challengingotherwise they would already have been determined in the current systems. In some countries, the adoption of the tool may also require changes in legislation. Better tools are indeed required for the reimbursement decision process, and the framework introduced is a commendable initiative. In its current form, the questions and the suggested criteria can be used as a useful basis by those involved in reimbursement decision making. Considering answers to the questions may help to piece together different, sometimes conflicting, aspects and sum them up as an opinion. For this reason alone, the work done by the authors is most welcome.
Jaana E. Martikainen

Head of Drug Research, Ph.D. (Pharm.)

The Social Insurance Institution, Research Department, Helsinki, Finland

e-mail: jaana.martikainen@kela.fi

\section{CONFLICT OF INTEREST}

The author reports she has no potential conflicts of interest.

\section{REFERENCES}

1. Cleemput I, Franken M, le Polain M, Koopmanschap M. European drug reimbursement systems' legitimacy: Five-country comparison and policy tool. Int J Technol Assess Health Care. 2012;28:358-366.

2. Franken M, le Polain M, Cleemput I, Koopmanschap M. Similarities and differences between five European drug reimbursement systems. Int $J$ Technol Assess Health Care. 2012;28:349-357.

3. Godman B, Shrank W, Andersen M, et al. Policies to enhance prescribing efficiency in Europe: Findings and future implications. Front Pharmacol. 2011;1:1-16

4. Martikainen J, Kivi I, Linnosmaa I. European prices of newly launched reimbursable pharmaceuticals - a pilot study. Health Policy. 2005;74:235246.

5. OECD. Health at a glance 2011. OECD indicators. OECD Publishing, 2011. http://www.oecd.org/dataoecd/6/28/49105858.pdf (accessed July 12, 2012).

6. Stolk P, Belitser SV, Leufkens HGM, Heerdink ER. Variable access to clopidogrel in a harmonized EU market. Value Health. 2008;11:989-995. 